\title{
Filtered Pseudo-parallel Corpus Improves Low-resource Neural Machine Translation
}

\author{
AIZHAN IMANKULOVA, TAKAYUKI SATO, and MAMORU KOMACHI, \\ Tokyo Metropolitan University, Japan
}

\begin{abstract}
Large-scale parallel corpora are essential for training high-quality machine translation systems; however, such corpora are not freely available for many language translation pairs. Previously, training data has been augmented by pseudo-parallel corpora obtained by using machine translation models to translate monolingual corpora into the source language. However, in low-resource language pairs, in which only low-accurate machine translation systems can be used, translation quality degrades when a pseudo-parallel corpus is naively used. To improve machine translation performance with low-resource language pairs, we propose a method to effectively expand the training data via filtering the pseudo-parallel corpus using quality estimation based on sentence-level round-trip translation. For experiments with three language pairs that utilized small, medium, and large size parallel corpora, BLEU scores significantly improved for low-resource language pairs. Additionally, the effects of iterative bootstrapping on translation performance quality is investigated; resultingly, it is confirmed that bootstrapping can further improve the translation performance.
\end{abstract}

CCS Concepts: • Computing methodologies $\rightarrow$ Machine translation;

Additional Key Words and Phrases: Pseudo-parallel corpus, filtering, low-resource language pairs, round-trip translation, sentence-level similarity metrics, bootstrapping

\section{ACM Reference format:}

Aizhan Imankulova, Takayuki Sato, and Mamoru Komachi. 2019. Filtered Pseudo-parallel Corpus Improves Low-resource Neural Machine Translation. ACM Trans. Asian Low-Resour. Lang. Inf. Process. 19, 2, Article 24 (October 2019), 16 pages.

https://doi.org/10.1145/3341726

\section{INTRODUCTION}

A large-scale parallel corpus is an essential resource for training statistical machine translation (SMT) and neural machine translation (NMT) systems. Creating a high-quality large-scale parallel corpus requires time, financial resources, and expert translation of a large amount of text. Resultingly, many existing large-scale parallel corpora are limited to specific languages and domains. Contrastingly, large monolingual corpora are easier to obtain.

Various approaches have been proposed to create a pseudo-parallel corpus from a monolingual corpus. For example, Zhang and Zong (2016) proposed a method to generate a pseudo-parallel corpus based on a monolingual corpus of the source language and its automatic translation. Sennrich

Authors' addresses: A. Imankulova, T. Sato, and M. Komachi, Tokyo Metropolitan University, Graduate School of System Design, 6-6 Asahigaoka, Hino, Tokyo, 191-0065, Japan; emails: imankulova-aizhan@ed.tmu.ac.jp, sasatatata99@gmail.com, komachi@tmu.ac.jp.

Permission to make digital or hard copies of all or part of this work for personal or classroom use is granted without fee provided that copies are not made or distributed for profit or commercial advantage and that copies bear this notice and the full citation on the first page. Copyrights for components of this work owned by others than ACM must be honored. Abstracting with credit is permitted. To copy otherwise, or republish, to post on servers or to redistribute to lists, requires prior specific permission and/or a fee. Request permissions from permissions@acm.org.

(C) 2019 Association for Computing Machinery.

2375-4699/2019/10-ART24 \$15.00

https://doi.org/10.1145/3341726 
et al. (2016) obtained substantial improvements by automatically translating a monolingual corpus of the target language into the source language, referred to as synthetic source sentences, and treating the obtained pseudo-parallel corpus as additional training data. They used monolingual data of the target language to more effectively learn the conditional language model. However, they experimented on language pairs for which relatively large-scale parallel corpora are available. Thus, they did not fully exploit the training corpus or address the quality of the pseudo-parallel corpus.

To address the quality of the parallel corpus, we propose a method to create a pseudo-parallel corpus by translating a monolingual corpus in the target language and filtering it using round-trip translation. If the target sentence and its round-trip translation are similar, then we assume that the synthetic source sentence is appropriate with respect to its monolingual target sentence; moreover, this pair can be included in the filtered pseudo-parallel corpus. The quality of the pseudoparallel corpus is particularly important because low-quality parallel sentences will degrade NMT performance more than SMT (Koehn and Knowles 2017). Accordingly, our motivation is to filter out low-quality synthetic sentences that might be included in such a pseudo-parallel corpus to obtain a high-quality pseudo-parallel corpus for low-resource language pairs. Filtration can be iteratively applied using an upgraded NMT system. Thus, the size of a high-quality pseudo-parallel corpus can be increased. To the best of our knowledge, this study is the first attempt to (1) filter a pseudo-parallel corpus using round-trip translation and (2) bootstrap NMT.

The main contributions of our research are as follows:

- To establish a high-quality pseudo-parallel corpus, we filter a pseudo-parallel corpus using three sentence-level similarity metrics: sentence-level Bilingual Evaluation Understudy Score (sent-BLEU) (Lin and Och 2004a, 2004b), average alignment similarity (AAS), and maximum alignment similarity (MAS) (Song and Roth 2015). We also use a sentence-level language model (sent-LM) to filter a pseudo-parallel corpus by only considering synthetic source sentences.

- We observe that bootstrapping using our proposed filtering method significantly improves the BLEU score; however, the gains in BLEU decrease gradually over several iterations.

- We show that the proposed filtering method, along with bootstrapping, is useful for lowresource language pairs.

- We publicly released the obtained filtered pseudo-parallel corpora. ${ }^{1}$

Here, we conducted experiments on three different language pairs that have varying amount of available parallel data. Japanese $\leftrightarrow$ Russian was used as the low-resource language pair, French $\rightarrow$ Malagasy as medium-resource language pair, and German $\rightarrow$ English as the high-resource language pair. We demonstrated that the baseline method (Sennrich et al. 2016) is effective for highresource language pairs; however, in the case of low-resource language pairs, it is more effective to use a filtered pseudo-parallel corpus as additional training data. With the filtered pseudo-parallel corpus, up to 3.46 BLEU points were achieved in the Russian $\rightarrow$ Japanese translation, and up to 5.25 BLEU points in the Japanese $\rightarrow$ Russian translation. This is in contrast to the baseline's -0.05 and 0.27 BLEU points, respectively.

The remainder of this article is organized as follows: Section 2 discusses previous studies related to improving low-resource machine translation systems; Section 3 outlines the proposed method for filtering a pseudo-parallel corpus and bootstrapping NMT; Sections 4 and 5 present our evaluation of the proposed model; and Section 6 describes the results. Conclusions and directions for future work are presented in Section 7.

$\overline{{ }^{1} \text { https://github.com/aizhanti/filtered-pseudo-parallel-corpora. }}$ 


\section{RELATED WORK}

To address data sparsity in machine translations, many methods use monolingual data to improve translation quality (Bertoldi and Federico 2009; Edunov et al. 2018; Hsieh et al. 2013; Schwenk 2008; Ueffing et al. 2007; Zhang and Zong 2016; Zhang et al. 2018). Specifically, Bertoldi and Federico (2009) addressed the problem of domain adaptation by training a translation model from a generated pseudo-parallel corpus created from a monolingual in-domain corpus. Hsieh et al. (2013) created a pseudo-parallel corpus from patterns learned from source and monolingual target indomain corpora for cross-domain adaptation. They manually conducted filtration of "relatively more accurate" translated sentences and used them to revise the language model. Several methods use iterative approaches to improve NMT using pseudo-parallel corpora (Cotterell and Kreutzer 2018; Hoang et al. 2018). Zhang et al. (2018) used both source and target monolingual corpora to iteratively improve an NMT system. Edunov et al. (2018) improved high-resource NMT using synthetic sources generated by sampling or adding noise to beam outputs. Imamura et al. (2018) sampled multiple sources for each target sentence to enhance the encoder and attention mechanism, leading to an improvement of translation quality. However, experiments were conducted on relatively high-resource language pairs. Niu et al. (2018) improved bi-directional NMT by continuously training on augmented parallel data. Similarly, in this study, we used a pseudo-parallel corpus created by translating a monolingual corpus from the target language rather than from the source language. Contrastingly, automatic filtering is applied to the obtained pseudo-parallel corpus. We conducted experiments on low-, medium-, and high-resource language pairs to demonstrate the accuracy of the filtered pseudo-parallel corpora created by the NMT.

Data filtering is often used in domain adaptation (Axelrod et al. 2011; Moore and Lewis 2010) for phrase-based SMT systems. Sentences are extracted from large corpora to optimize the language model and the translation model (Wang et al. 2014; Yildız et al. 2014). The work most closely related to our study is that of Ylldiz et al. (2014), in which a quality estimator was built to obtain high-quality parallel sentence pairs using a bilingual dictionary. They achieved improved translation performance and reduced the time complexity with a small high-quality corpus. This method filters data by calculating the similarity between the source and target sentences. In our work, the similarity is calculated between monolingual and synthetic target sentences without relying on any external dictionaries.

Recently, van der Wees et al. (2017) performed dynamic data selection in the training of an NMT model. To sort and filter the training data, they used language models from the source and target sides of in-domain and out-of-domain data to calculate cross-entropy scores. However, in the present study, round-trip translation is employed to filter data while taking into consideration their meaning.

Meanwhile, He et al. (2016) presented a dual-learning approach. It simultaneously trains two models through a reinforcement learning process. Monolingual data of both source and target languages are used, and informative feedback signals are generated to train the translation models. The dual-learning approach was shown to alleviate the issue of noisy data by increasing their quality. In our approach, however, we attempt to remove noisy data. In addition, He et al. (2016) assumed a high-recourse language pair to "warm-start" the reinforcement learning process, whereas we target low-resource language pairs, wherein high-quality seed NMT models are difficult to obtain.

A completely unsupervised approach (Artetxe et al. 2018; Lample et al. 2018) has been useful in a zero-shot scenario by exploiting only monolingual corpora and back-translation. Nevertheless, we focus on maximizing the utility of existing small parallel corpora, leaving the application of recent unsupervised MT methods for future work. 


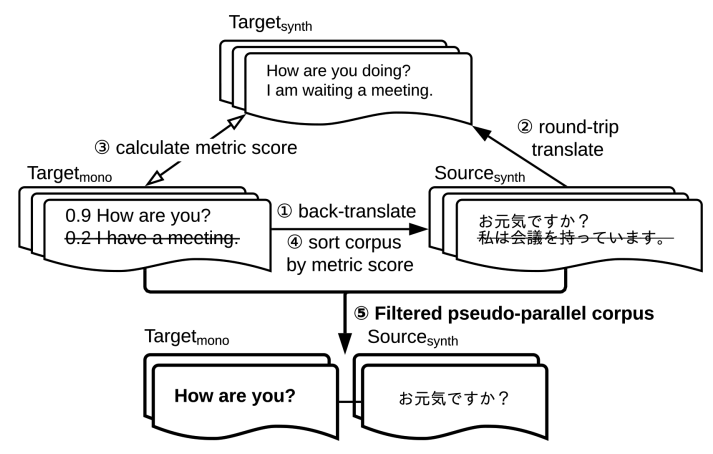

Fig. 1. Creating and filtering a pseudo-parallel corpus using round-trip translation.

This work is a non-trivial extension of Imankulova et al. (2017). Our previous work focused on only two filtering metrics: sent-BLEU for all language pairs and sent-LM only for the Russian $\rightarrow$ Japanese language pair. Here, our experiments are presented with two additional sentence similarity metrics for filtering: AAS and MAS (see Section 3.3). While our previous work reported the effect of bootstrapping only on the Russian $\rightarrow$ Japanese language pair, this study investigates the effect of bootstrapping on multiple language pairs. Additionally, all models were trained 10 times with different seeds to reduce training variance and reported the scores of the best model on the development set.

\section{IMPROVING LOW-RESOURCE NMT WITH FILTERED PSEUDO-PARALLEL CORPUS}

The proposed method involves filtering a pseudo-parallel corpus by round-trip translation of a monolingual corpus for low-resource language pairs. Then, by iterating the filtering process until convergence, bootstrapping an NMT model is attempted.

The overall algorithm is described in Algorithms 1 and 2.

\subsection{Filtering}

As shown in Figure 1, the proposed method includes the following steps:

(1) Back-translate monolingual target sentences (Target ${ }_{\text {mono }}$ ) using a "Model ${ }_{b}$ " model trained in the target $\rightarrow$ source direction to produce synthetic source sentences (Source synth $_{\text {). Here, }}$ an "Unfiltered" pseudo-parallel corpus is obtained as additional data without filtration, similar to the approach used in Sennrich et al. (2016).

(2) Round-trip translate the synthetic source sentences using a "Model $l_{f}$ " model trained in the source $\rightarrow$ target direction to obtain a synthetic target sentence $\left(\right.$ Target $\left._{\text {synth }}\right)$.

(3) Calculate sentence-level similarity metric scores using the monolingual target sentences as a reference and the synthetic target sentences as candidates.

(4) Sort the monolingual target sentences and the corresponding synthetic source sentences in a descending order of sentence-level similarity metric scores and filter out sentences with low scores. The threshold is determined by the translation quality on the development set.

(5) Use the filtered synthetic source sentences as the source side and the monolingual target sentences as the target side of the pseudo-parallel corpus; this is referred to as a "Filtered" pseudo-parallel corpus: It is used as training data in addition to the parallel corpus to train a new "Filtered" model. 


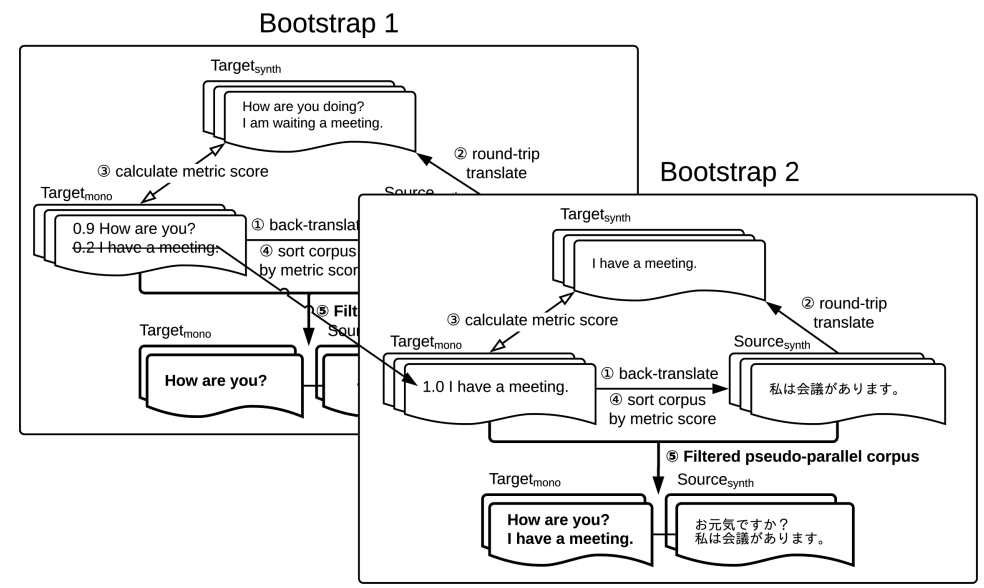

Fig. 2. Bootstrapping NMT with a pseudo-parallel corpus.

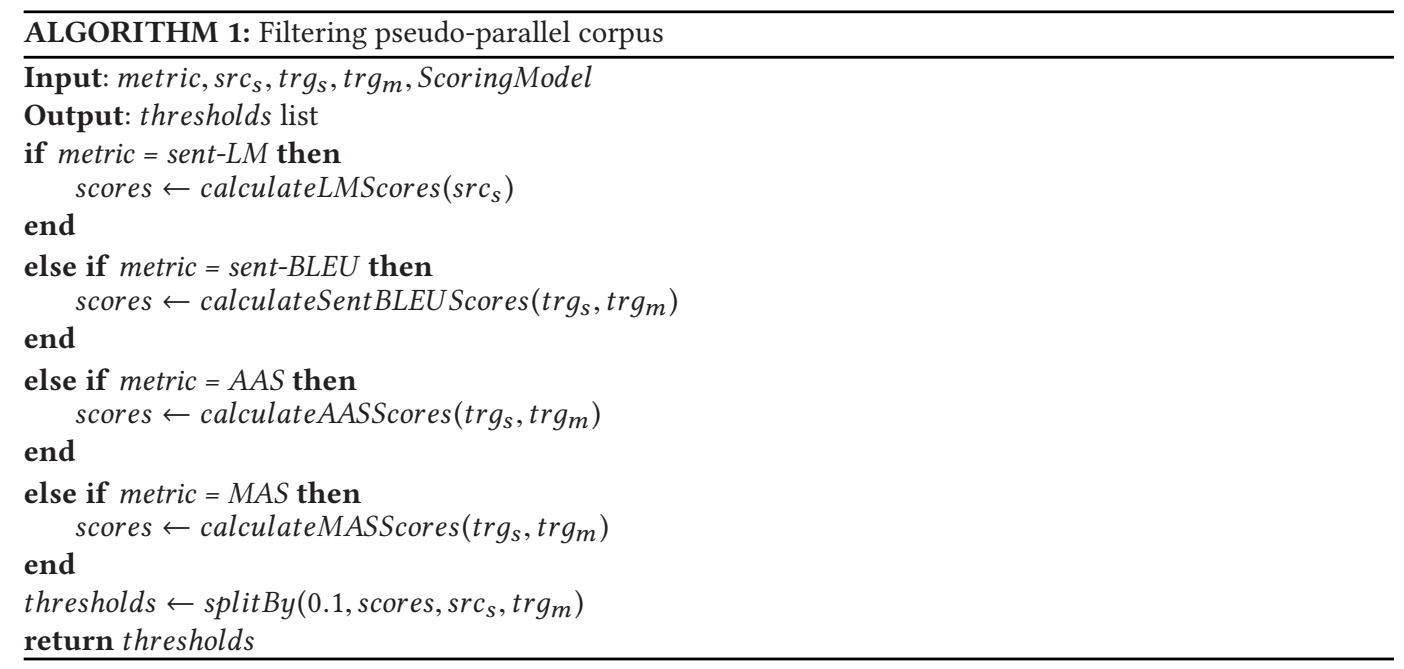

\subsection{Bootstrapping}

Each bootstrapping iteration involves the following steps (Figure 2):

(1) "Model training": Train "Filtered" NMT models using a parallel corpus and additional "Filtered" pseudo-parallel corpora created by the proposed filtering method.

(2) "Model selection": Select the best model on the development set from the previous iteration and use it instead of the source $\rightarrow$ target "Model $f$ " model from the previous iteration. Additionally, train its target $\rightarrow$ source "Model $b$ " model.

(3) "Bootstrapping": Use target sentences from the pseudo-parallel corpus that have been filtered out from training data of the previous best model to create new "Filtered" pseudoparallel corpora to bootstrap the NMT. If there is no improvement over the previous iteration, then terminate the bootstrapping process and return to the "Filtered"pseudo-parallel corpus and the translation model as output.

(4) Repeat steps 1 to 3. 


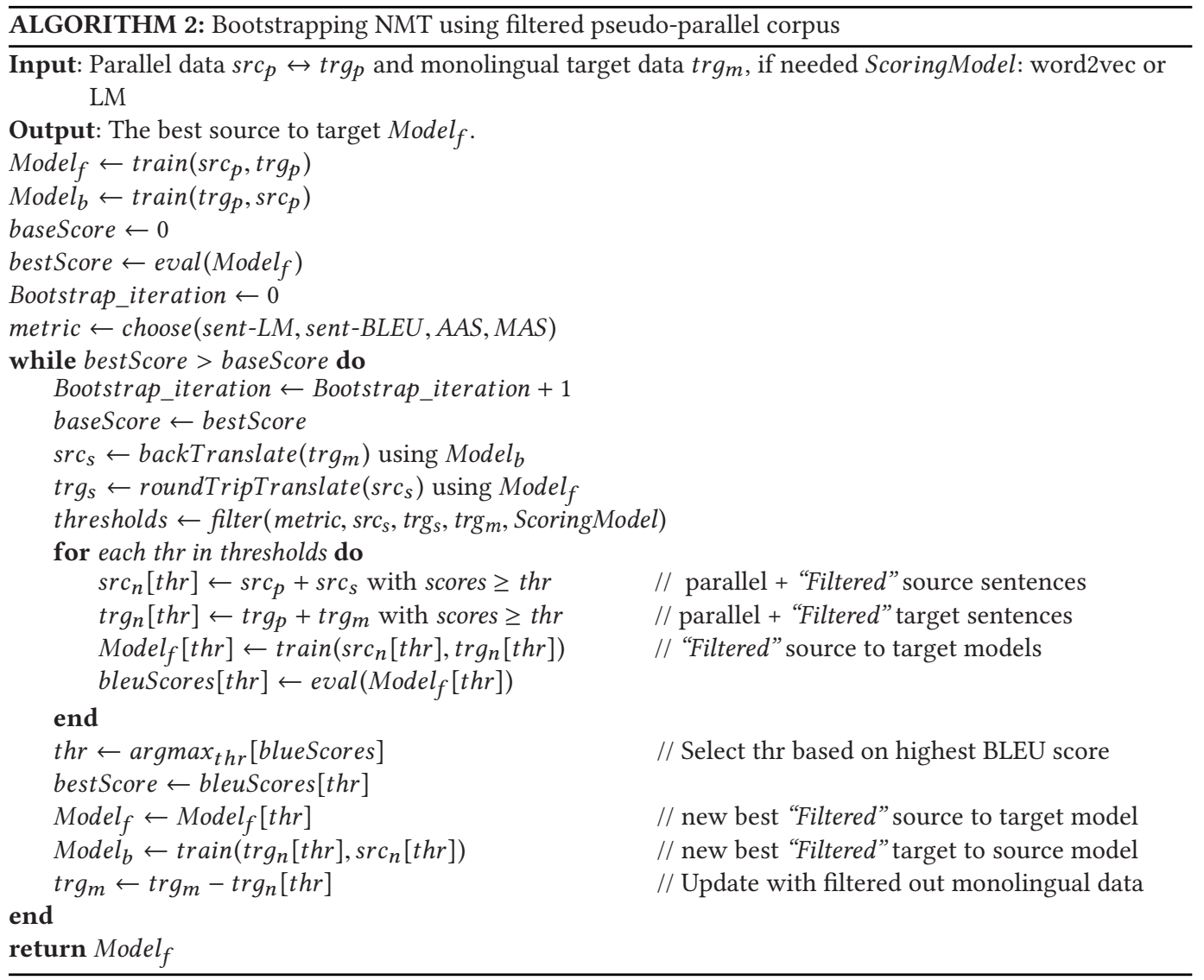

To create a new pseudo-parallel corpus for a new bootstrap iteration, we use those monolingual target sentences that were not included in the "Filtered" pseudo-parallel corpus of the previous iteration. Consequently, the already created "Filtered" pseudo-parallel corpus from the previous iteration does not change ${ }^{2}$ in the next bootstrap iteration. Even if the filtered-out monolingual target sentences remain the same, its synthetic source sentences are refreshed at each iteration. Thus, the translation quality of both the "Unfiltered" and "Filtered" pseudo-parallel corpus is improved via the bootstrapping process until the stopping criterion is met.

\subsection{Sentence-level Similarity Metrics for Filtering}

Three sentence-level similarity metrics are used for filtering: sent-BLEU, AAS, and MAS proposed by Song and Roth (2015), which showed effective results in the Semantic Textual Similarity task (Kajiwara et al. 2017). These metrics require round-trip translation of target monolingual data for the proposed filtration method. Sent-BLEU calculates the similarity of the synthetic and monolingual target sentences based on only surface information, whereas AAS and MAS use distributed representations of the sentences.

\footnotetext{
${ }^{2}$ The attempt to update the entire pseudo-parallel corpus in each bootstrap iteration, instead of using only filtered-out monolingual data to create a new pseudo-parallel corpus for a new bootstrap iteration, led to degraded performance.
} 
The AAS score is the average cosine similarity between vectors of all words in monolingual and synthetic target sentences:

$$
\operatorname{AAS}\left(y, y^{\prime}\right)=\frac{1}{|y|\left|y^{\prime}\right|} \sum_{i=1}^{|y|} \sum_{j=1}^{\left|y^{\prime}\right|} \cos \left(\overrightarrow{y_{i}} \overrightarrow{y_{j}^{\prime}}\right) .
$$

The MAS score is the cosine similarity between the most similar word from the monolingual target sentence and each word from the synthetic target sentence:

$$
\operatorname{MAS}_{\text {asym }}\left(y, y^{\prime}\right)=\frac{1}{|y|} \sum_{i=1}^{|y|} \max _{j} \cos \left(\overrightarrow{y_{i}} \overrightarrow{y_{j}^{\prime}}\right) .
$$

Note that this similarity is not symmetric. A symmetric similarity can be computed by averaging two similarities:

$$
\operatorname{MAS}\left(y, y^{\prime}\right)=\frac{1}{2} \operatorname{MAS}_{\text {asym }}\left(y, y^{\prime}\right)+\frac{1}{2} \operatorname{MAS}_{\text {asym }}\left(y^{\prime}, y\right) \text {. }
$$

Here, $y=\left(y_{1}, \ldots, y_{i}\right)$ are word vectors for a monolingual target sentence, and $y^{\prime}=\left(y_{1}^{\prime}, \ldots, y_{j}^{\prime}\right)$ are word vectors for a synthetic target sentence.

\subsection{Sentence-level Language Model Scoring for Filtering}

We also used the sent-LM metric, which, in contrast to the other three sentence-level similarity metrics, performs filtration by scoring only synthetic source sentences without round-trip translation.

\subsection{Thresholds for the Pseudo-parallel Corpus}

Accordingly, the translation performance increases as the number of parallel sentences increases (Koehn 2002). However, for a pseudo-parallel corpus, the translation performance does not necessarily increase with the number of sentences. To determine the effects of the quantity and quality of the pseudo-parallel corpus in NMT, thresholds of metric scores are set with increment steps of 0.1 . Thus, pseudo-parallel sentences included as additional data have sentence-level similarity scores greater than or equal to some threshold (e.g., sent-BLEU $\geq 0.1, \ldots$, sent-BLEU $\geq 0.9, \ldots$ ). Sentences scored and filtered by filtering metrics were used to train the "Filtered" models. For example, sentences with filtering metric scores (e.g., sent-BLEU) greater than or equal to 0.1 were used to train the "sent-BLEU $\geq 0.1$ " model. Moreover, the NMT system was trained using different thresholds and their respective performances compared with development and test sets. Only source $\rightarrow$ target NMT models with the highest BLEU score on the development set were reported.

\section{EXPERIMENTS USING A FILTERED PSEUDO-PARALLEL CORPUS}

\subsection{Toolkits}

For the conducted experiments, we used the OpenNMT toolkit ${ }^{3}$ (Klein et al. 2017) to train all translation models. For the Russian $\leftrightarrow$ Japanese and French $\rightarrow$ Malagasy experiments, the following parameters were used: The number of recurrent layers of the encoder and decoder was one, BiLSTM was used with concatenation, the maximum batch size was 32 , and the Adadelta optimization method was applied. For the German $\rightarrow$ English experiments, OpenNMT default settings were used to match the hyper-parameters used for pre-trained German $\rightarrow$ English models (without back-translation) distributed by OpenNMT. ${ }^{4}$ The vocabulary size in all experiments was $50 \mathrm{~K}$.

\footnotetext{
${ }^{3}$ http://opennmt.net/OpenNMT/.

${ }^{4}$ http://opennmt.net/Models/.
} 
All French, English, German, and Russian sentences were tokenized and true-cased using Moses scripts. ${ }^{5}$ For all Japanese sentences, MeCab 0.996 was used with the IPAdic dictionary ${ }^{6}$ for word segmentation. For all languages, duplicate sentences and sentences with more than 50 words were eliminated. To compare the translation results, the BLEU scores (Papineni et al. 2002) were recorded. Additionally, Moses's bootstrap-hypothesis-difference-significance.pl script was used to perform statistical significance tests on the translations $(p<0.05)$.

The sent-BLEU scores were calculated using the MTeval-sentence of the MTeval toolkit. ${ }^{7}$ Word2vec (Mikolov et al. 2013) models were trained using the Gensim library to calculate AAS and MAS metrics. The KenLM Language Model Toolkit ${ }^{8}$ was used to build a 5-gram language model with Kneser-Ney smoothing. To extract the scores, the filtering metric scores were normalized to be between $[0,1]$ using a feature-scaling preprocessing.MinMaxScaler method from the scikit-learn library, which transforms features by scaling each feature within the designated min and max range. The LM log probability scores are normalized by dividing the log probability scores by the sentence length.

\subsection{Dataset}

4.2.1 Parallel and Target Monolingual Data. The parallel corpora for low-resource Russian $\leftrightarrow$ Japanese $^{9}$ and for medium-resource French $\rightarrow$ Malagasy $^{10}$ experiments were downloaded from OPUS. ${ }^{11}$ For the medium-resource French-Malagasy language pair, the news domain GlobalVoices corpus was used, which differs from the Tatoeba ${ }^{12}$ daily-conversations domain corpus used in the Russian $\leftrightarrow$ Japanese experiments. The GlobalVoices corpus has more available parallel data (107,406 sentence pairs compared with 11,231 available through Tatoeba).

The duplicate Tatoeba parallel corpus was divided for the Russian $\leftrightarrow$ Japanese experiments as follows: 10,231 sentences composed the training set, 500 sentences the development set, and 500 sentences the test set. Additionally, to perform Japanese $\rightarrow$ Russian $\rightarrow$ Japanese round-trip translation for the Russian-to-Japanese experiment, all available 165,742 Japanese monolingual sentences were sampled from in-domain Tatoeba. All available 75,402 Russian monolingual sentences from in-domain Tatoeba were also sampled for Japanese $\rightarrow$ Russian translation to facilitate Russian $\rightarrow$ Japanese $\rightarrow$ Russian round-trip translation. None of the utilized monolingual data overlapped with the parallel data.

Experiments for the French $\rightarrow$ Malagasy language pair were conducted with data from the GlobalVoices corpus. Parallel data were divided as follows: 106,406 sentences composed the training set, 1,000 sentences the development set, and 1,000 sentences the test set. From GlobalVoices, 105,573 Malagasy monolingual sentences were used to create a French $\rightarrow$ Malagasy pseudo-parallel corpus.

For the German $\rightarrow$ English experiments, pre-trained German $\leftrightarrow$ English models and 4,535,522 parallel sentences provided by OpenNMT were downloaded. The default OpenNMT settings were used to preprocess all data. 4,208,439 German $\rightarrow$ English sentences from automatically backtranslated monolingual data ${ }^{13}$ was downloaded; the synthetic German side was translated back to English using the pre-trained German $\rightarrow$ English model to filter this pseudo-parallel corpus. For

\footnotetext{
$\overline{{ }^{5} \text { https://github.com/moses-smt/mosesdecoder/. }}$

${ }^{6} \mathrm{http}: / /$ taku910.github.io/mecab.

${ }^{7}$ https://github.com/odashi/mteval.

${ }^{8}$ https://kheafield.com/code/kenlm/.

${ }^{9}$ http://opus.lingfil.uu.se/Tatoeba.php.

${ }^{10} \mathrm{http}$ ///opus.lingfil.uu.se/GlobalVoices.php.

${ }^{11}$ http://opus.lingfil.uu.se/.

${ }^{12}$ https://tatoeba.org/eng.

${ }^{13} \mathrm{http}: / /$ data.statmt.org/rsennrich/wmt16_backtranslations/de-en/.
} 
Table 1. Data Statistics

\begin{tabular}{|l|ccc|}
\hline Corpus & Russian $\leftrightarrow$ Japanese & French $\rightarrow$ Malagasy & German $\rightarrow$ English \\
\hline Train parallel & 10,231 & 106,406 & $4,535,522$ \\
Dev & 500 & 1,000 & 3,000 \\
Test & 500 & 1,000 & 3,003 \\
Monolingual target & $75,402 \leftrightarrow 165,742$ & 105,570 & $4,208,439$ \\
\hline
\end{tabular}

the development set, newtest2013 (3,000 sentence pairs) was used, and for the test set, newtest2014 (3,003 sentence pairs). Table 1 shows the data statistics.

4.2.2 Data to Train word2vec Models. To train word2vec models for the Russian $\leftrightarrow$ Japanese experiments, the OpenSubtitles 2018 corpus ${ }^{14}$ was chosen, as its domain is most similar to the Tatoeba domain, with high-resource data. Japanese and Russian monolingual OpenSubtitles2018 corpora were downloaded from OPUS. ${ }^{15}$ After tokenizing and removing sentences with less than 2 and more than 100 words, 2,728,314 Japanese monolingual sentences were obtained to train Japanese word2vec. Similarly, Russian monolingual sentences were cleaned and the same 2,728,314 sentences sampled to match Japanese the monolingual data, for fair comparison on low-resource settings. To train the Malagasy word2vec model for the French $\rightarrow$ Malagasy experiments, data was used from the Leipzig Corpora Collection for Under-resourced Languages ${ }^{16}$ (Goldhahn et al. 2016) and from GlobalVoices for all Malagasy monolingual data. The total size of the employed data was 296,440 Malagasy monolingual sentences. For the German $\rightarrow$ English experiments, the English side of parallel and monolingual data used in the previous Section 4.2.1 was concatenated, resulting in 8,743,962 English monolingual sentences.

4.2.3 Data to Train Language Models. To train the language models for the Japanese $\rightarrow$ Russian and Russian $\rightarrow$ Japanese experiments, the same Japanese and Russian sentences were used that were used to train the word2vec models. For the French $\rightarrow$ Malagasy experiments, 2,190,579 French monolingual sentences were used from the Europarl. ${ }^{17}$ For the German $\rightarrow$ English experiments, German monolingual sentences were downloaded from automatically back-translated data ${ }^{18}$ and concatenated with the German side of parallel data, resulting in 8,115,406 German monolingual sentences.

\subsection{Baselines}

Sennrich et al. (2016) obtained additional training data by automatically translating monolingual target sentences into the source language with their "Parallel" baseline systems. Our process differs from theirs in that we construct "Parallel" baseline machine translation systems in both directions using an available parallel corpus to generate a filtered pseudo-parallel corpus.

The baseline systems used herein were (1) the "Parallel" systems trained on a parallel corpus in both directions, which were then used to create a pseudo-parallel corpus, and (2) an "Unfiltered" system, which was trained on a concatenated parallel corpus with all pseudo-parallel corpora without any filtration.

\footnotetext{
${ }^{14}$ http://opus.nlpl.eu/OpenSubtitles2018.php/.

${ }^{15}$ http://opus.nlpl.eu/index.php.

${ }^{16} \mathrm{http}: / /$ curl.corpora.uni-leipzig.de/languages/mlg.

${ }^{17}$ http://www.statmt.org/wmt14/training-monolingual-europarl-v7/europarl-v7.fr.gz.

${ }^{18} \mathrm{http} / / /$ data.statmt.org/rsennrich/wmt16_backtranslations/en-de/.
} 
Table 2. Russian $\rightarrow$ Japanese Translation BLEU Scores

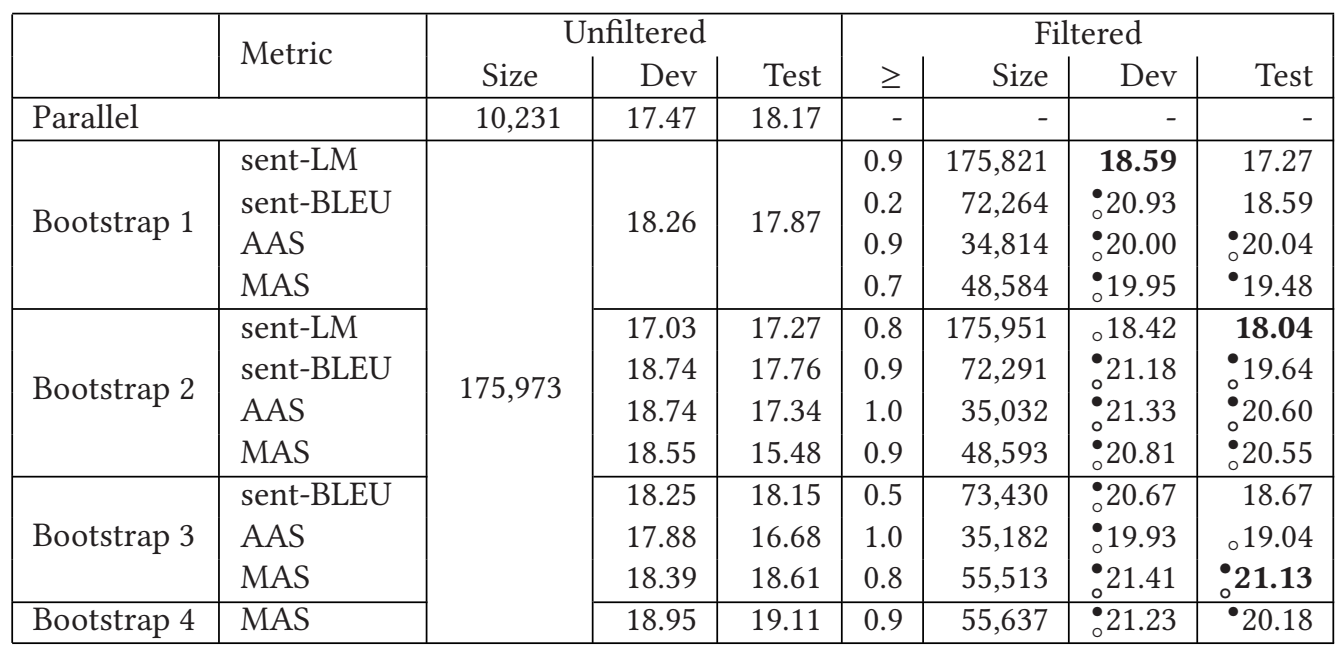

There is a statistically significant difference for ${ }^{\bullet}$ : against the "Parallel" system, and for $\circ$ : against the "Unfiltered" system of that Bootstrap iteration. Bold indicates the highest BLEU scores for each filtering metric.

\subsection{Filtering and Bootstrapping}

The parallel sentence pairs (Section 4.2) were used to train the baseline "Parallel" models in both directions for all language pairs. Then, these models were used to create a pseudo-parallel corpus by round-trip translating the target monolingual sentences (Section 3). A concatenation of parallel and pseudo-parallel sentences was used to train the "Unfiltered" models for each bootstrapping iteration. Because of training variance, all "Unfiltered" models were trained 10 times with different seeds, as well as one time for each threshold; the scores of the best model on the development set is then reported. For each filtering metric, after filtration and selection of the best model on the development set and its data, the filtered-out target monolingual sentences are used for round-trip translation with the chosen model. Since different models are used, "Unfiltered" data is created with different quality for each filtering metrics. Here, for each filtering metric, the BLEU scores are compared for the development sets. Iterating was halted if the current score was lower than the previous socre. Accordingly, the BLEU scores for development and test sets (Tables 2-5) for "Parallel," "Unfiltered," and "Filtered" models with their data sizes and thresholds (columns " $\geq$ ") are reported.

\section{RESULTS}

In this section, the results on three different language pairs are described: Japanese $\leftrightarrow$ Russian, French $\rightarrow$ Malagasy, and German $\rightarrow$ English.

\subsection{Russian $\rightarrow$ Japanese}

Table 2 outlines the results of all bootstrap iterations and filtering metrics for the Russian $\rightarrow$ Japanese language pair. The results obtained using the "Unfiltered" model demonstrate that none of the "Unfiltered" models significantly outperformed the "Parallel" model.

Contrastingly, "Filtered" models significantly outperformed both "Parallel" and their "Unfiltered" baselines. In most cases, the difference between the BLEU scores of "Filtered" and "Parallel" on the development set are around +3 points. Generally, these results suggest that using filtered 
Table 3. Japanese $\rightarrow$ Russian Translation BLEU Scores

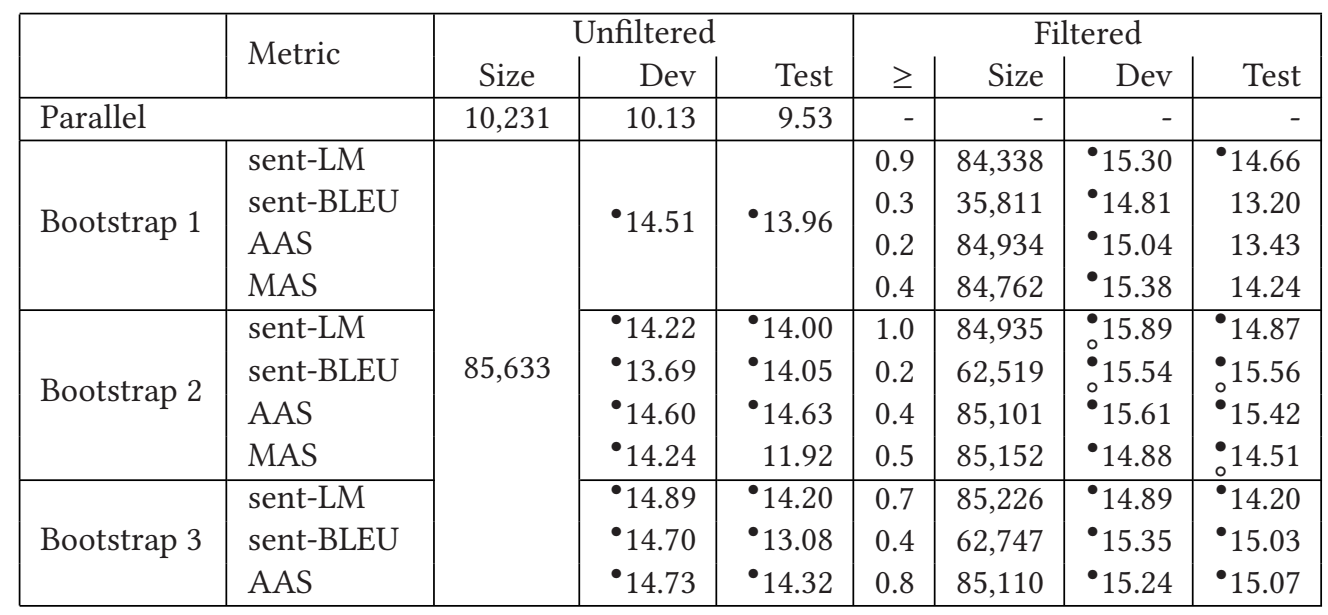

There is a statistically significant difference, for ': against the "Parallel" system, and for $\circ$ : against the "Unfiltered" system of that Bootstrap iteration. Bold indicates the highest BLEU scores for each filtering metric.

pseudo-parallel data rather than all sentences containing incorrect sentence pairs leads to increased machine translation accuracy for both baselines.

The model trained using data scored by a sent-LM metric stopped improving after the first bootstrap iteration. However, using sentence-level similarity metrics significantly increased the performance over baselines, even when much fewer data were used for the training. The best "MAS $\geq 0.8$ " model outperformed the "Parallel" and its "Unfiltered" model in terms of BLEU scores on the development set by +3.94 and +3.02 points, respectively.

\subsection{Japanese $\rightarrow$ Russian}

The effect of the proposed filtering method on Japanese $\rightarrow$ Russian translations was examined, with the results given in Table 3. Most of the "Unfiltered" models performed significantly better than the "Parallel" model.

However, all "Filtered" models still returned better BLEU scores than the "Parallel" and "Unfiltered" models. The difference between the BLEU scores of the "Filtered" and "Parallel" models on development and test sets are generally more than +4 points. The "sent- $L M \geq 1.0$ " model in Bootstrap 2 outperformed the "Parallel" and its "Unfiltered" model in terms of BLEU scores on the development set by +5.76 and +1.67 points, respectively. However, its BLEU score on the test set was low compared to other metrics' models. In Bootstrap 3, The BLEU scores of "sent-LM $\geq 0.7$ " were the same as that of its "Unfiltered" model. We assume that the relatively weak models from the previous iteration generated low-quality pseudo-parallel data, which led to weaker "Filtered" models that used nearly the same amount of data as the "Unfiltered" model.

\subsection{French $\rightarrow$ Malagasy}

The results for French $\rightarrow$ Malagasy are shown in Table 4. Here, only some of the "Unfiltered"models slightly outperformed the "Parallel" model. Despite the fact that "Filtered" models showed higher BLEU scores than both baselines, only some showed significant improvements. On the development set, the best "AAS $\geq 0.9$ " model from Bootstrap 2 yielded better results up to +0.99 BLEU points and +0.46 BLEU points over the "Parallel" and "Unfiltered" baselines, respectively. 
Table 4. French $\rightarrow$ Malagasy Translation BLEU Scores

\begin{tabular}{|c|c|c|c|c|c|c|c|c|}
\hline & \multirow{2}{*}{ Metric } & \multicolumn{3}{|c|}{ Unfiltered } & \multicolumn{4}{|c|}{ Filtered } \\
\hline & & Size & Dev & Test & $\geq$ & Size & Dev & Test \\
\hline \multicolumn{2}{|l|}{ Parallel } & 106,406 & 16.78 & 15.18 & - & - & - & - \\
\hline \multirow{4}{*}{ Bootstrap 1} & sent-LM & \multirow{10}{*}{211,979} & & & 0.8 & 211,823 & $\bullet 17.44$ & 15.72 \\
\hline & sent-BLEU & & 1706 & 1499 & 0.3 & 124,756 & $\bullet 17.43$ & 14.39 \\
\hline & AAS & & 17.00 & 14.90 & 0.9 & 175,386 & $\bullet 17.59$ & $\bullet 16.87$ \\
\hline & MAS & & & & 0.7 & 210,851 & $\bullet 17.43$ & 15.67 \\
\hline \multirow{4}{*}{ Bootstrap 2} & sent-LM & & 17.00 & 16.06 & 0.9 & 211,949 & $\bullet 17.35$ & 15.65 \\
\hline & sent-BLEU & & 16.69 & 15.32 & 0.6 & 125,176 & •17.69 & 15.71 \\
\hline & AAS & & 17.31 & 15.35 & 0.9 & 189,929 & $\bullet 17.77$ & $\bullet 16.26$ \\
\hline & MAS & & 16.80 & 15.52 & 0.5 & 211,840 & $\bullet 17.38$ & 16.06 \\
\hline \multirow{2}{*}{ Bootstrap 3} & sent-BLEU & & 16.87 & 16.03 & 0.9 & 125,185 & 17.19 & 15.64 \\
\hline & AAS & & 17.07 & $\bullet 16.21$ & 0.8 & 204,817 & $\bullet 17.52$ & 15.62 \\
\hline
\end{tabular}

There is a statistically significant difference, for ${ }^{\bullet}$ : against the "Parallel" system, and for $。$ : against the "Unfiltered" system of that Bootstrap iteration. Bold indicates the highest BLEU scores for each filtering metric.

Table 5. German $\rightarrow$ English Translation BLEU Scores

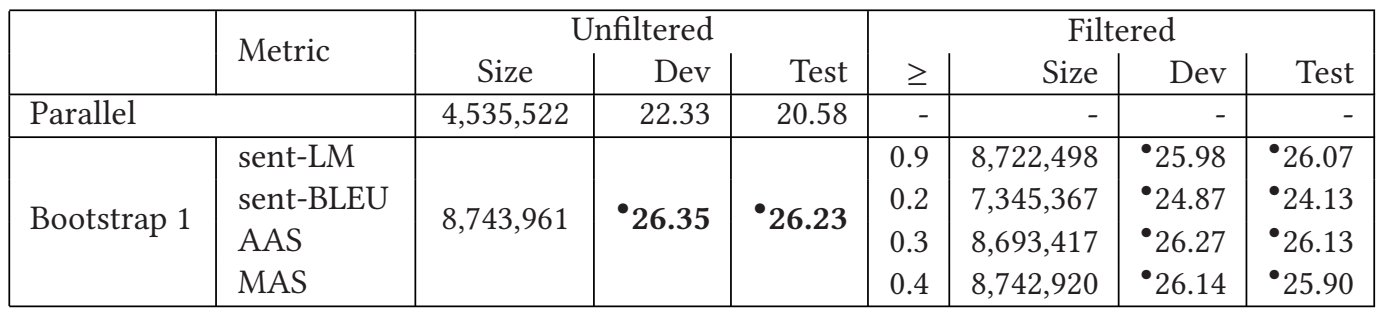

There is a statistically significant difference, for $\bullet$ : against the "Parallel" system, and for ${ }_{\circ}$ : against the "Unfiltered" system of that Bootstrap iteration. Bold indicates the highest BLEU scores for each filtering metric.

We assume that since French $\rightarrow$ Malagasy "Parallel" models were trained using medium-resource data, they generated a pseudo-parallel corpus with better quality than low-resource language pairs. Even though the filtration method improved baseline models, improvements were not as significant as in low-resource scenarios.

\subsection{German $\rightarrow$ English}

Table 5 shows the BLEU scores of German $\rightarrow$ English experiments. All models that used additional pseudo-parallel corpora achieved significant improvements over the "Parallel" baseline. However, none of the "Filtered" models outperformed the "Unfiltered" baseline on the development and test sets, regardless of filtering metrics.

We assume, in this case, that the "Parallel" models were strong enough to generate a large amount of high-quality pseudo-parallel sentences. Thus, using all pseudo-parallel corpus without any filtration improved the "Parallel" baseline the most. Additionally, filtering out some noisy pseudo-parallel sentences resulted in weaker models than in the "Unfiltered" baseline.

\subsection{Human Evaluation}

Additionally, a pairwise method was utilized for the human evaluation of Russian $\rightarrow$ Japanese translation of the four bootstrap models created using the MAS metric. Two human evaluators were asked to evaluate the translations of 100 source sentences randomly sampled from the test set. 
Table 6. Human Evaluation of Russian $\rightarrow$ Japanese

Translation Adequacy and Fluency

of the Bootstrapped Models

\begin{tabular}{|l|c|c|c|}
\hline Iteration & Model & Adequacy & Fluency \\
\hline Bootstrap 1 & MAS $\geq 0.7$ & 21 & 56 \\
Bootstrap 2 & MAS $\geq 0.9$ & 16 & 43 \\
Bootstrap 3 & MAS $\geq 0.8$ & $\mathbf{2 2}$ & $\mathbf{5 7}$ \\
Bootstrap 4 & MAS $\geq 0.9$ & 17 & 48 \\
\hline
\end{tabular}

Each evaluator chose the best candidate based on adequacy and fluency without knowing which bootstrap system produced the respective translation (ties and "none" were allowed). The final decision was made by the voting. The number of times each bootstrap model was selected as the best translation by the evaluators was then calculated.

Table 6 shows the results of the human evaluation. The highest number of correct answers for adequacy and fluency was obtained by the "MAS $\geq 0.8$ " model in Bootstrap 3. Thus, bootstrapping had a positive effect on the NMT model.

\section{DISCUSSION}

The results of all experiments showed that, rather than using all additional pseudo-parallel data, the proposed filtering method improved the translation performance in nearly all experiments conducted for low-resource language pairs. The extensive experiments using four different filtering metrics showed that filtering itself significantly impacts low-resource language pairs, as the improvements across different filtering metrics were consistent with the insignificant differences.

Table 7 shows examples of Russian $\rightarrow$ Japanese pseudo-parallel sentences scored by the MAS metric for every bootstrapping iteration. In the first example, the synthetic Russian sentence from Bootstrap 1 was an incorrect translation of its Japanese monolingual sentence. However, by Bootstrap 3, the synthetic Russian and its synthetic Japanese translation improved. By the final Bootstrap 4 iteration, the translation output was sufficient. Finally, these synthetic Russian and Japanese monolingual sentences were used to train the best "MAS $\geq 0.9$ " model from Bootstrap 4. Accordingly, the Japanese $\rightarrow$ Russian and Russian $\rightarrow$ Japanese models filtered by MAS metric used to create the pseudo-parallel corpora improved with each bootstrap iteration. Example 2 shows that the synthetic Russian sentence from Bootstrap 3 was an adequate translation of its Japanese monolingual sentence, whereas its round-trip translation was not. As a result, these pseudo-parallel sentences were filtered out, resulting in a slightly worse synthetic Russian sentence, in terms of adequacy, in Bootstrap 4. Therefore, such noisy sentences are still present in the filtered pseudo-parallel corpus used as additional training data for the best model, which led to the decreased translation quality of the Russian $\rightarrow$ Japanese NMT model.

Sennrich et al. (2016) showed that using a pseudo-parallel corpus as additional data greatly improved the performance over the "Parallel" baseline. Furthermore, our experiments demonstrated that for a high-resource language pair (Table 5), the proposed filtering method was unable to improve the performance over the "Unfiltered" baseline; additionally, it was not as effective as it was for a low-resource language pair. The experiments showed that a better "Parallel" system resulted in the creation of a better pseudo-parallel corpus. Thus, the weaker the "Parallel" system, the more effective the proposed filtration method.

For some filtration metrics in the experiments of Tables 2-4, removing only very few of the training data improved BLEU scores over the "Unfiltered" baselines. After manual inspection, 
Table 7. Examples from the Russian $\rightarrow$ Japanese Pseudo-parallel Corpus Scored by the MAS Metric and Their Changes on Every Bootstrapping Iteration

\begin{tabular}{|c|c|c|c|}
\hline Boot & Synthetic Russian Sentence & Synthetic Japanese Sentence & MAS score \\
\hline \multicolumn{4}{|c|}{ Example 1 - Japanese Monolingual Sentence: ミルクを一杯いかが。 (how about a cup of milk ?) } \\
\hline Bootstrap 1 & $\begin{array}{l}\text { не кофе, пожалуйста . } \\
\text { (no coffee, please .) }\end{array}$ & $\begin{array}{l}\text { コ-ヒーをください。 } \\
\text { (coffee, please .) }\end{array}$ & 0.63 \\
\hline Bootstrap 2 & $\begin{array}{l}\text { не кофе , пожалуйста . } \\
\text { (no coffee, please .) }\end{array}$ & $\begin{array}{l}\text { コ-ヒ-を飲んでください。 } \\
\text { (please, drink coffee.) }\end{array}$ & 0.54 \\
\hline Bootstrap 3 & $\begin{array}{l}\text { не могли ли чашку молока? } \\
\text { (couldn't a cup of milk ?) }\end{array}$ & $\begin{array}{l}\text { 牛乳を1杯いただけませんか。 } \\
\text { (could I have a glass of milk?) }\end{array}$ & 0.67 \\
\hline Bootstrap 4 & $\begin{array}{l}\text { не хотите ли чашку молока ? } \\
\text { (would you like a cup of milk?) }\end{array}$ & $\begin{array}{l}\text { ミルクを一杯いかがですか。 } \\
\text { (would you like a cup of milk?) }\end{array}$ & 0.92 \\
\hline \multicolumn{4}{|c|}{$\begin{array}{r}\text { Example } 2 \text { - Japanese Monolingual Sentence:トムはメアリーについてあなたに話しましたか。 } \\
\text { (did Tom talk to you about Mary ?) }\end{array}$} \\
\hline Bootstrap 1 & $\begin{array}{l}\text { Том говорил, что ты говорил ? } \\
\text { (Tom said what did you say ?) }\end{array}$ & $\begin{array}{l}\text { トムは何を言っているのだと言ったんだ? } \\
\text { (did you say what Tom is saying?) }\end{array}$ & 0.64 \\
\hline Bootstrap 2 & $\begin{array}{l}\text { Том говорил с Вами? } \\
\text { (did Tom talk to you ?) }\end{array}$ & $\begin{array}{l}\text { トムはあなたと話しましたか。 } \\
\text { (did Tom talk to you ?) }\end{array}$ & 0.76 \\
\hline Bootstrap 3 & $\begin{array}{l}\text { Том говорил с Вами по Мэри . } \\
\text { (Tom spoke to you by Mary .) }\end{array}$ & $\begin{array}{l}\text { トムは一人であなたに会った。 } \\
\text { (Tom saw you by yourself .) }\end{array}$ & 0.74 \\
\hline Bootstrap 4 & $\begin{array}{l}\text { Том говорил с Вами? } \\
\text { (did Tom talk to you ?) }\end{array}$ & $\begin{array}{l}\text { トムはあなたに話しましたか。 } \\
\text { (did Tom tell you ?) }\end{array}$ & 0.91 \\
\hline
\end{tabular}

the removed data were particularly noisy. For example, in Bootstrap 1 of Table 3 for the Japanese $\rightarrow$ Russian experiments, "sent- $L M \geq 0.9$ " removed pairs with a score of 0.55 as follows:

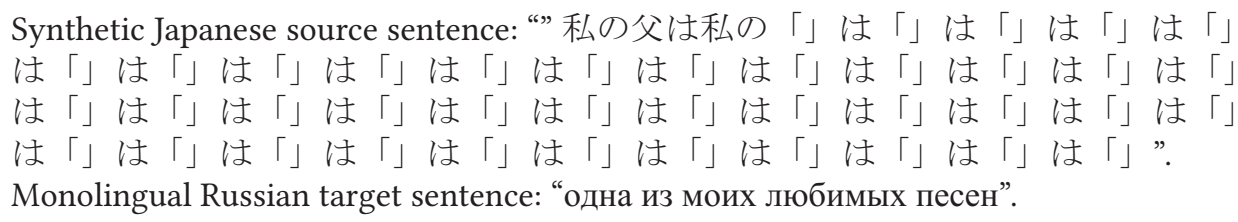

We assume that such noisy data greatly affects already weak NMT quality, especially in lowresource scenarios; the NMT system "unlearns" its conditioning on the source context when the training data are noisy. This phenomenon could be addressed as the low-resource case of the work by Sennrich et al. (2016) with their "Dummy Source Sentences" experiments. It is also known that in low-resource settings, neural networks tend to experience overfitting (Srivastava et al. 2014). Considering the number of parameters, NMT systems tend to overfit on small training data: i.e., they learn both the correct and the noisy information from the given data. The "Unfiltered" models are greatly influenced by the training data with respect to the noisy data removed by filtering. However, with filtering, sentences containing such noise can be successfully removed, leading to the increased BLEU scores. From this, the utility of filtering is established: The noisy data can be correctly removed from the experiment settings when systems are susceptible to the training data.

The experimental results showed that bootstrapping for multiple iterations improved NMT in terms of the BLEU score. However, the quality ceases to improve after several steps. This could be attributed to the systems used to create new pseudo-parallel corpora, which become weaker at each bootstrap iteration. 
The low-resource NMT systems depend not only on the amount of training data, but also on the quality of the data. Therefore, even if the number of removed sentences is relatively small, it is more appropriate to define the threshold as an absolute value, rather than a percentage of the data, because the result may change significantly.

\section{CONCLUSION}

Advanced pseudo-parallel corpora filtration methods were proposed that employ round-trip translation of a target monolingual corpus and bootstrapping to obtain high-quality pseudo-parallel corpora for low-resource language pairs. The models trained using the filtered pseudo-parallel corpus as additional data returned a better translation performance than did the baselines for lowresource language pairs; none of the filtered models outperformed the "Unfiltered" baseline for the high-resource language pair. Additionally, the translation performance can be further improved by bootstrapping, although bootstrapping has its limitations with regard to the BLEU score. These findings suggest that translation accuracy depends on both the size and quality of the training data. The weaker the "Parallel" system used in the creation of a pseudo-parallel corpus, the lower the quality of the created pseudo-parallel corpus. In this scenario, the proposed filtration method can be the most useful for obtaining an improved pseudo-parallel corpus.

Further experimental investigations are required to estimate the limitations of the proposed filtration method. In this work, three different language pairs were considered, wherein each contained a different amount of available parallel data with different domains. To extend this research, we plan to investigate the effect of the proposed filtration method with the same language domains and sizes of parallel data; we believe that involving source monolingual data would further improve NMT using our proposed filtration method. We also plan to improve our filtering method by estimating the quality of synthetic translations with reinforcement learning.

\section{REFERENCES}

Mikel Artetxe, Gorka Labaka, Eneko Agirre, and Kyunghyun Cho. 2018. Unsupervised neural machine translation. In Proceedings of the International Conference on Learning Representations.

Amittai Axelrod, Xiaodong He, and Jianfeng Gao. 2011. Domain adaptation via pseudo in-domain data selection. In Proceedings of the Conference on Empirical Methods in Natural Language Processing. 355-362.

Nicola Bertoldi and Marcello Federico. 2009. Domain adaptation for statistical machine translation with monolingual resources. In Proceedings of the 4th Workshop on Statistical Machine Translation. 182-189.

Ryan Cotterell and Julia Kreutzer. 2018. Explaining and generalizing back-translation through wake-sleep. Retrieved from: CoRR abs/1806.04402 (2018).

Sergey Edunov, Myle Ott, Michael Auli, and David Grangier. 2018. Understanding back-translation at scale. In Proceedings of the Conference on Empirical Methods in Natural Language Processing. 489-500.

Dirk Goldhahn, Maciej Sumalvico, and Uwe Quasthoff. 2016. Corpus collection for under-resourced languages with more than one million speakers. In Proceedings of the Workshop on Collaboration and Computing for Under-Resourced Languages (CCURL'16), Language Resources and Evaluation Conference. 67-73.

Di He, Yingce Xia, Tao Qin, Liwei Wang, Nenghai Yu, Tieyan Liu, and Wei-Ying Ma. 2016. Dual learning for machine translation. In Proceedings of the Conference on Advances in Neural Information Processing Systems. 820-828.

Vu Cong Duy Hoang, Philipp Koehn, Gholamreza Haffari, and Trevor Cohn. 2018. Iterative back-translation for neural machine translation. In Proceedings of the 2nd Workshop on Neural Machine Translation and Generation. 18-24.

An-Chang Hsieh, Hen-Hsen Huang, and Hsin-Hsi Chen. 2013. Uses of monolingual in-domain corpora for cross-domain adaptation with hybrid MT approaches. In Proceedings of the 2nd Workshop on Hybrid Approaches to Translation. $117-$ 122 .

Kenji Imamura, Atsushi Fujita, and Eiichiro Sumita. 2018. Enhancement of encoder and attention using target monolingual corpora in neural machine translation. In Proceedings of the 2nd Workshop on Neural Machine Translation and Generation. $55-63$.

Aizhan Imankulova, Takayuki Sato, and Mamoru Komachi. 2017. Improving low-resource neural machine translation with filtered pseudo-parallel corpus. In Proceedings of the 4th Workshop on Asian Translation. 70-78. 
Tomoyuki Kajiwara, Danushka Bollegala, Yuichi Yoshida, and Ken-ichi Kawarabayashi. 2017. An iterative approach for the global estimation of sentence similarity. PloS ONE 12, 9 (2017), e0180885.

Guillaume Klein, Yoon Kim, Yuntian Deng, Jean Senellart, and Alexander M. Rush. 2017. OpenNMT: Open-source toolkit for neural machine translation. Retrieved from: arXiv preprint arXiv:1701.02810.

Philipp Koehn. 2002. Europarl: A Multilingual Corpus for Evaluation of Machine Translation. http://homepages.inf.ed.ac. uk/pkoehn/publications/europarl.pdf.

Philipp Koehn and Rebecca Knowles. 2017. Six challenges for neural machine translation. In Proceedings of the 1st Workshop on Neural Machine Translation. 28-39.

Guillaume Lample, Alexis Conneau, Ludovic Denoyer, and Marc'Aurelio Ranzato. 2018. Unsupervised machine translation using monolingual corpora only. In Proceedings of the International Conference on Learning Representations.

Chin-Yew Lin and Franz Josef Och. 2004a. Automatic evaluation of machine translation quality using longest common subsequence and skip-bigram statistics. In Proceedings of the 42nd Meeting on Association for Computational Linguistics. 605-612.

Chin-Yew Lin and Franz Josef Och. 2004b. Orange: A method for evaluating automatic evaluation metrics for machine translation. In Proceedings of the 20th International Conference on Computational Linguistics. 501-507.

Tomas Mikolov, Kai Chen, Greg Corrado, and Jeffrey Dean. 2013. Efficient estimation of word representations in vector space. In Proceedings of the International Conference on Learning Representations.

Robert C. Moore and William Lewis. 2010. Intelligent selection of language model training data. In Proceedings of the Meeting on Association for Computational Linguistics Conference Short Papers. 220-224.

Xing Niu, Michael Denkowski, and Marine Carpuat. 2018. Bi-directional neural machine translation with synthetic parallel data. In Proceedings of the 2nd Workshop on Neural Machine Translation and Generation. 84-91.

Kishore Papineni, Salim Roukos, Todd Ward, and Wei-Jing Zhu. 2002. BLEU: A method for automatic evaluation of machine translation. In Proceedings of the 40th Meeting on Association for Computational Linguistics. 311-318.

Holger Schwenk. 2008. Investigations on large-scale lightly-supervised training for statistical machine translation. In Proceedings of International Workshop on Spoken Language Translation. 182-189.

Rico Sennrich, Barry Haddow, and Alexandra Birch. 2016. Improving neural machine translation models with monolingual data. In Proceedings of the 54th Meeting of the Association for Computational Linguistics (Volume 1: Long Papers). 86-96.

Yangqiu Song and Dan Roth. 2015. Unsupervised sparse vector densification for short text similarity. In Proceedings of the Conference of the North American Chapter of the Association for Computational Linguistics: Human Language Technologies. 1275-1280.

Nitish Srivastava, Geoffrey E. Hinton, Alex Krizhevsky, Ilya Sutskever, and Ruslan Salakhutdinov. 2014. Dropout: A simple way to prevent neural networks from overfitting. J. Machine Learn. Res. 15, 1 (2014), 1929-1958.

Nicola Ueffing, Gholamreza Haffari, and Anoop Sarkar. 2007. Transductive learning for statistical machine translation. In Proceedings of the 45th Meeting of the Association of Computational Linguistics. 25-32.

Marlies van der Wees, Arianna Bisazza, and Christof Monz. 2017. Dynamic data selection for neural machine translation. In Proceedings of the Conference on Empirical Methods in Natural Language Processing. 1400-1410.

Longyue Wang, Derek F. Wong, Lidia S. Chao, Yi Lu, and Junwen Xing. 2014. A systematic comparison of data selection criteria for SMT domain adaptation. The Scientific World fournal. 1-10.

Eray Yıldız, Ahmed Cüneyd Tantuğ, and Banu Diri. 2014. The effect of parallel corpus quality vs size in English-to-Turkish SMT. In Proceedings of the 6th International Conference on Web services and Semantic Technology. 21-30.

Jiajun Zhang and Chengqing Zong. 2016. Exploiting source-side monolingual data in neural machine translation. In Proceedings of the Conference on Empirical Methods in Natural Language Processing. 1535-1545.

Zhirui Zhang, Shujie Liu, Mu Li, Ming Zhou, and Enhong Chen. 2018. Joint training for neural machine translation models with monolingual data. In Proceedings of the AAAI Conference on Artificial Intelligence. 555-562.

Received April 2018; revised April 2019; accepted June 2019 